\title{
AN ANALYSIS OF THE CONTACT BETWEEN THE STENT AND THE ARTERY USING TUBE HIDROFORMING SIMULATION
}

\author{
R. Araújo ${ }^{1}$, S. A. G. Oliveira ${ }^{1}$, T. A. Guimarães ${ }^{2}$ \\ ${ }^{1}$ Faculty of Mechanical Engineering, Federal University of Uberlândia (corresponding- \\ rogerau_mat@yahoo.com.br)
}

${ }^{2}$ Department of Mathematics, Federal University of Goiás, Campus Catalão

\begin{abstract}
Stents for angioplasty have been extensively used in the treatment of the coronary diseases. After the angioplasty the expanded stent in contact with the arterial wall should avoid restenosis or re-closure of the artery. On the other hand, the contact stress between the stent and the artery may cause hyperplasia and restenosis due to the vascular injury. Therefore, the objective of this work is to study the expansion of the stent and the contact with the artery using the tube hidroforming simulation. In the simulation, it will be studied the contact stress and the final shape of the artery caused by the stent expansion process using the Stampack software, an explicit finite elements code. In this work, it was used a geometrical model of a commercial stent made of 316 L stainless steel. The stent material was modeled by using an elastic-plastic constitutive law with isotropic hardening. The artery was modeled as a cylinder and its material is hyperelastic. The results showed that the methodology has proposed in this work may be used for checking if the stent model implanted in the artery may cause restenosis after the angioplasty.
\end{abstract}

Keywords: Angioplasty, Explicit finite elements method, Tube hidroforming, Stents.

\section{INTRODUCTION}

Stents for angioplasty are devices that are implanted in the arterial wall in order to avoid the restenosis [1]. After the angioplasty, the expanded stent should have high radial strength to prevent the re-closure or the vessel recoil. Hence, most of commercial stent are geometrically designed to support the pressure has imposed by the arterial wall. However, a stiffer stent may also cause damages to artery when subjected to the angioplasty [2]. The vascular injury due to the implantation of a stent may cause hyperplasia and eventual restenosis. Recently, drugs-eluting stents have been successfully used in patients in the prevention of the hyperplasia and restenosis of the arterial wall. Another way of avoiding the restenosis is to decrease the contact stress level in the arterial wall caused by the stent expansion process after the angioplasty [2]. 
Liang et al. [3] used the finite elements analysis in order to study the interaction between the stent and a plaque placed on the arterial wall. They used a Von-Mises constitutive law for the material model of the stent and a viscoelastic material model for the plaque. Prendergast et al. [2] also used the finite element method to investigate the effect of the different geometrical models of stents on the contact stress level caused in the arterial wall after the angioplasty. In this case, they employed an elastic-plastic Von Mises constitutive law for modeling the material of the stent and a hyper-elastic model for the material of the artery and the plaque. In both studies, it was demonstrated that the geometrical model of the stent may cause large contact stress in the arterial wall and hyperplasia after the angioplasty. A stent with a large thickness may increase the restenosis rate in patients. Furthermore, the ends of the stent and the flexible link elements in its structure may also increase the risk of hyperplasia.

In this work, it will be studied the contact stress and the final shape of plaque, artery and stent using the finite elements simulation. For the simulation, it will be used the hidroforming module available in the Stampack program [4]. This explicit finite elements software is commonly used in the analysis and the design of the metallic sheets subjected to the forming process. Another objective is to check the structural integrity of the artery in the expansion process simulation using the Forming Limit Diagram and the presence of wrinkling, thinning and buckling in the stent structure. On this way, it will be possible to predict if there are rupture risk in the stent material and the artery.

\section{MATERIALS AND METHODS}

\subsection{Simulation of expansion process of the stent using tube hidroforming}

Tube hidroforming is a manufacturing process that uses internal pressure applied by a fluid medium in order to obtain tubular components with desired a cross-section [5]. Recently, this manufacturing process has been largely used in the forming of automotive components, as for example, exhaust parts, radiator frames, etc. In this work, it will be used tube hidroforming simulation by finite elements has adapted to the angioplasty procedure, since the stent expansion process is totally analogous to this forming process.

The figure 1 illustrates the geometrical model of the stent. The figure 2 shows the stent inside the artery and plaque before the finite elements simulation. Initially, the stent geometrical model was developed in the AutoCAD software. Subsequently, this stent model was imported and meshed in the Stampack software. The explicit finite elements approach from Stampack software is more computationally eficient when compared to the implicit approach. Another advantage of this method is that the strain and stress field of the stent and artery is computed from the boundary conditions and no contact algorithm is required in the analysis.

The table 1 describes the geometrical parameters values of the stent, artery and plaque used in the finite elements analysis. In the simulation, the dimensions of the devices were amplified by a factor of 100 . This amplification of the size of stent, artery and plaque was necessary in order to decrease the processing time in the angioplasty procedure simulation. Since 
the parameters have studied in the post-processing stage from Stampack program are the plastic strain and the final shape, this increasing of the devices dimensions does not have influence in the results analysis [6].

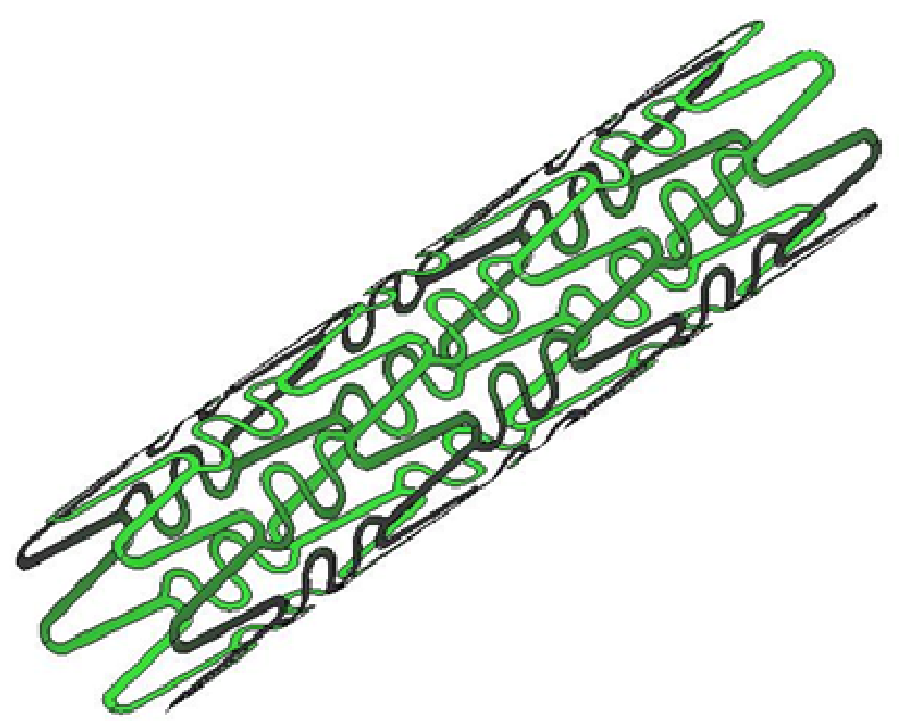

Figure 1. Stent geometrical model used in the tube hidroforming simulation.

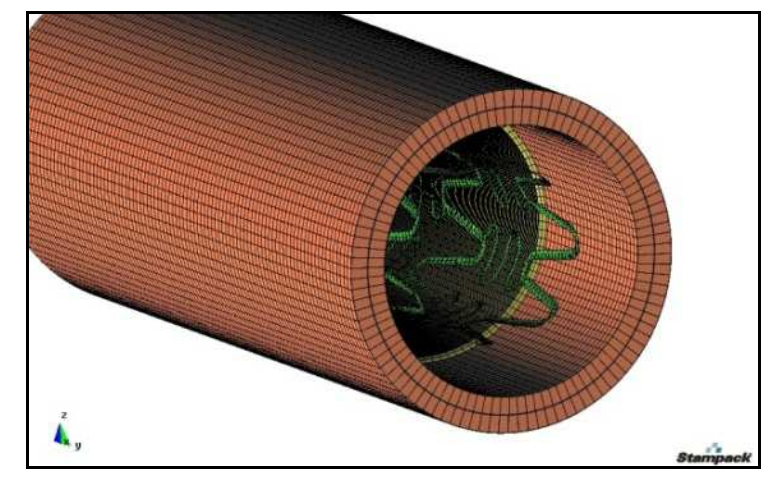

Figure 2. Finite elements models of the stent, artery and stenotic plaque.

Table 1. Geometrical parameters of the stent, artery and fat plaque.

\begin{tabular}{c|c|c|c|c|c}
\hline Device $\downarrow$ & $\begin{array}{c}\text { Dimensions } \\
\rightarrow\end{array}$ & $\mathrm{L}[\mathrm{mm}]$ & $\mathrm{h}[\mathrm{mm}]$ & $\mathrm{d}_{\mathrm{i}}[\mathrm{mm}]$ & $\mathrm{d}_{\mathrm{e}}[\mathrm{mm}]$ \\
\hline \multicolumn{2}{c|}{ stent } & 1317 & 10 & 300 & 310 \\
artery & 1500 & 60 & 450 & 570 \\
plaque & 732,6 & $18-50$ & $400-432$ & 450 \\
\hline
\end{tabular}

$\mathrm{L}=$ length $\mathrm{h}=$ thickness; $\mathrm{d}_{\mathrm{i}}=$ internal diameter; $\mathrm{d}_{\mathrm{e}}=$ external diameter; 


\subsection{Finite elements models of the stent, artery and plaque}

The stent geometrical model showed in the Fig.1 was meshed using triangular shell finite elements with three nodes [7]. Because of the geometrical complexity of the stent design, this type of finite element is more appropriated for the generation of its unstructured meshing. Another advantage of shell element is the reduction of the processing time using the explicit finite elements analysis [7]. After the stent meshing, its geometrical model has 21596 elements.

The artery and the plaque geometrical models illustrated in the Fig. 1 were meshed using volumetric finite elements with 8 nodes. In this case, both models were generated by employing a structured meshing. For the simulation of the angioplasty process and contact of the stent with the artery and plaque, it was applied an outward pressure in the internal surface of the stent. In this work, it will not be considered the presence expandable balloon in the angioplasty procedure simulation. The total time of the expansion simulation was $1.5 \times 10^{-3} \mathrm{~s}$. This value was considered based on the results of a typical angioplasty [8]. Moreover, all nodes from finite elements model are free to move in any direction.

The outward pressure magnitude applied on the internal surface of the stent was computed based on the trial-and-error. One of the objectives of the angioplasty simulation in the present paper is to increase the stent diameter in about 50\%. In the practice, it is difficult to predict the pressure value which will cause this amplification of the stent diameter, since the angioplasty simulation is highly nonlinear. For the simulation, the pressure will change linearly with the time since the zero value until the magnitude adjusted by trial-and-error.

\subsection{Materials models of the stent, artery and plaque}

Most of papers have published in the literature on the angioplasty procedure simulation use implicit finite elements codes based on the incremental approach for computing the plastic stress and strain field in the stent and artery [2], [3], [9]. Usually, the internal pressure applied in the expandable balloon is considered static and the dynamics effects of loading with the time are negligible in the analysis. Indeed, the stent expansion simulation using the explicit finite elements approach is an initial value and boundary conditions problem. Moreover, since the plastic strain distribution of the stent is changing with the time, the Von Mises constitutive law used in the modeling of stent material could not provide the correct results. Therefore, a more appropriate constitutive law for the stent material should be considered in the dynamic stress analysis during the angioplasty process.

The stent model has considered in this work is made of $316 \mathrm{~L}$ stainless steel. In most papers have found in the literature on the angioplasty process simulation the stent material is modeled as a Von Mises constitutive law with isotropic hardening. In this paper, since the angioplasty procedure will be simulated using tube hydroforming process, it will be applied a more appropriated material model for the stent forming. In this case, the Stampack software uses a more general material law [4]: 


$$
\sigma_{e q}^{2}=\sigma_{11}^{2}+\frac{r_{0}\left(1+r_{90}\right)}{r_{90}\left(1+r_{0}\right)} \sigma_{22}^{2}-2 \frac{r_{0}}{1+r_{0}} \sigma_{11} \sigma_{22}+\frac{\left(1+r_{45}\right)\left(r_{0}+r_{90}\right)}{r_{90}\left(1+r_{0}\right)} \sigma_{12}^{2} .
$$

where the $\sigma_{11}$ and $\sigma_{22}$ denotes the principal normal stress and $\sigma_{12}$ the shearing stress. The variables $r_{0}, r_{45}$ and $r_{90}$, known as Lankford parameters, define the plastic material anisotropy angles in degrees. The subscripts 0,45 and 90 represent the angles in degrees of the anisotropy directions from material. Since the stent material is isotropic, all anisotropy parameters are equal to 1 . In this way, it can be demonstrated that the equation (1) reduced to the Von Mises constitutive law for the equivalent stress, $\sigma_{\text {eq }}$.

In the incremental finite elements codes, usually the plastic range of the stent material is modeled by a linear or multi-linear approximation in the the strain and stress diagram [3], [4]. This model is more used in the stent expansion analysis when subjected to a static pressure which is not dependent on the time. For the angioplasty procedure by using the tube hydroforming simulation, the outward pressure applied on the internal surface from changes with respect to the time. In this case, the Ludwig-Nadai law is more appropriated for modeling of the stent material:

$$
\sigma_{e q}=k\left(\varepsilon_{p o}+\varepsilon_{p}\right)^{n}
$$

where $\mathrm{k}, \mathrm{n}$ and $\varepsilon_{\mathrm{p}}$ are parameters of the stent material to be experimentally determined in a uniaxial tensile test of a $316 \mathrm{~L}$ stainless steel piece. The parameter $\varepsilon_{\mathrm{po}}$ and $\varepsilon_{\mathrm{p}}$ are the effective plastic strain and the initial plastic strain, respectively. The Equation (2) is a more used material model for the the sheets forming analysis by finite elements [4]. The parameters of this model for the stent material are described in the Table 2.

Table 2. Parameters from the Ludwig-Nadai model for the stent material (available in the Stampack program).

\begin{tabular}{l|l}
\hline & \multicolumn{2}{c}{ Parameters } \\
\hline Elasticity Modulus & $183 \mathrm{GPa}$ \\
Poisson ratio & 0.29 \\
Density & $8030 \mathrm{~kg} / \mathrm{m}^{3}$ \\
$\mathrm{n}$ & 0.28 \\
$\mathrm{k}$ & $1160.4 \mathrm{MPa}$ \\
$\mathrm{R}_{0,45,90}$ & 1 \\
$\varepsilon_{\mathrm{po}}$ & 0.21 \\
\hline
\end{tabular}

During the angioplasty procedure, the artery and plaque material are subjected to the large strains in the elastic range due to the stent expansion process. However, the strain-stress relationship for the artery and plaque is usually non-linear. Therefore, most of papers have published in the literature on analysis contact stress between the stent and the artery employ a 
hyperelastic constitutive law for the modeling of the artery and plaque material [2], [9]. In the present paper, it will be used the Ogden Model as the artery and plaque material constitutive law. In this model, the strain energy density of the artery and plaque material, $\mathrm{W}$, is given by [10]:

$$
W=\sum_{i=1}^{N} \frac{\mu_{i}}{\alpha_{i}}\left(\lambda_{1}^{-\alpha_{i}}+\lambda_{2}^{-\alpha_{i}}+\lambda_{3}^{-\alpha_{i}}-3\right)
$$

where $\lambda_{1}, \lambda_{2}$ and $\lambda_{3}$ are the principal stretch ratios of the material and $\mu_{\mathrm{i}}$ and $\alpha_{\mathrm{i}}$ are parameters from Ogden model. The variable $\mathrm{N}$ defines the order from Ogden model. The tables 3 and 4 describe the value of these parameters for the stenotic plaque and arterial tissue material respectively [10].

By using the Eq. (3), the stress components are obtained by differentiating the strain energy, W, with respect to the strain components. For the modeling of the tissue material constitutive law from plaque and artery, the order from model will be equal to 3 (three). The hyperelastic Mooney-Rivlin model could also be used as the plaque and artery material model [2], [9]. Neverthless, the Stampack program employs this constitutive law model for the tube material in the hydroforming process.

Table 3. Parameters of the Ogden's model for the arterial tissue material [10].

\begin{tabular}{l|l}
\hline Ogden parameters & \\
\hline$\alpha 1$ & 21.83 \\
$\mu 1$ & $-13[\mathrm{MPa}]$ \\
$\alpha 2$ & 22.22 \\
$\mu 2$ & $7.9[\mathrm{MPa}]$ \\
$\alpha 3$ & 21.15 \\
$\mu 3$ & $5.1[\mathrm{MPa}]$ \\
\hline
\end{tabular}

Table 4. Parameters of the Ogden's model for the stenotic plaque material [10].

\begin{tabular}{l|l}
\hline Ogden parameters & \\
\hline$\alpha 1$ & 2 \\
$\mu 1$ & $-4.6[\mathrm{MPa}]$ \\
$\alpha 2$ & 4 \\
$\mu 2$ & $3.23[\mathrm{MPa}]$ \\
$\alpha 3$ & -1.99 \\
$\mu 3$ & $1.6[\mathrm{MPa}]$ \\
\hline
\end{tabular}




\section{ANALYSIS OF THE RESULTS}

After the numerical simulation of the angioplasty process using the finite elements, the final shape and the stress and strain field in the stent, artery and plaque were analyzed in the post-processing step from Stampack software. For the stent expansion, it was applied a dynamic pressure on its internal surface with magnitude equals to the $4.2 \times 10^{7} \mathrm{~N} / \mathrm{m}^{2}$ during $1.5 \times 10^{-3} \mathrm{~s}$. This pressure value was obtained by trial-and-error after some simulations of the angioplasty process.

The figure 3 illustrates the final shape and the plastic strain distribution in the stent after the expansion. For the pressure value equals to $4.2 \times 10^{-7} \mathrm{~N} / \mathrm{m}^{2}$, the diameter increased $52 \%$ at the ends from stent. In the middle region, the stent diameter increased $37 \%$ approximately. In this way, the final diameters from stent model in amplified scale are equal to $458 \mathrm{~mm}$ and $413 \mathrm{~mm}$, respectively. It is interesting to observe that the ends of the stent are more deformed when compared to the middle region. This excessive strain from stent may cause damages in the arterial wall [2]. Therefore, the artery may be subjected to hiperplasia and restenosis after the angioplasty procedure. On the other hand, the elastic recoil of the stent length after the expansion was of $1.24 \%$. Since this value is relatively small, a possible lesion placed in the arterial wall would be totally supported by the implantation of the stent in the angioplasty process.

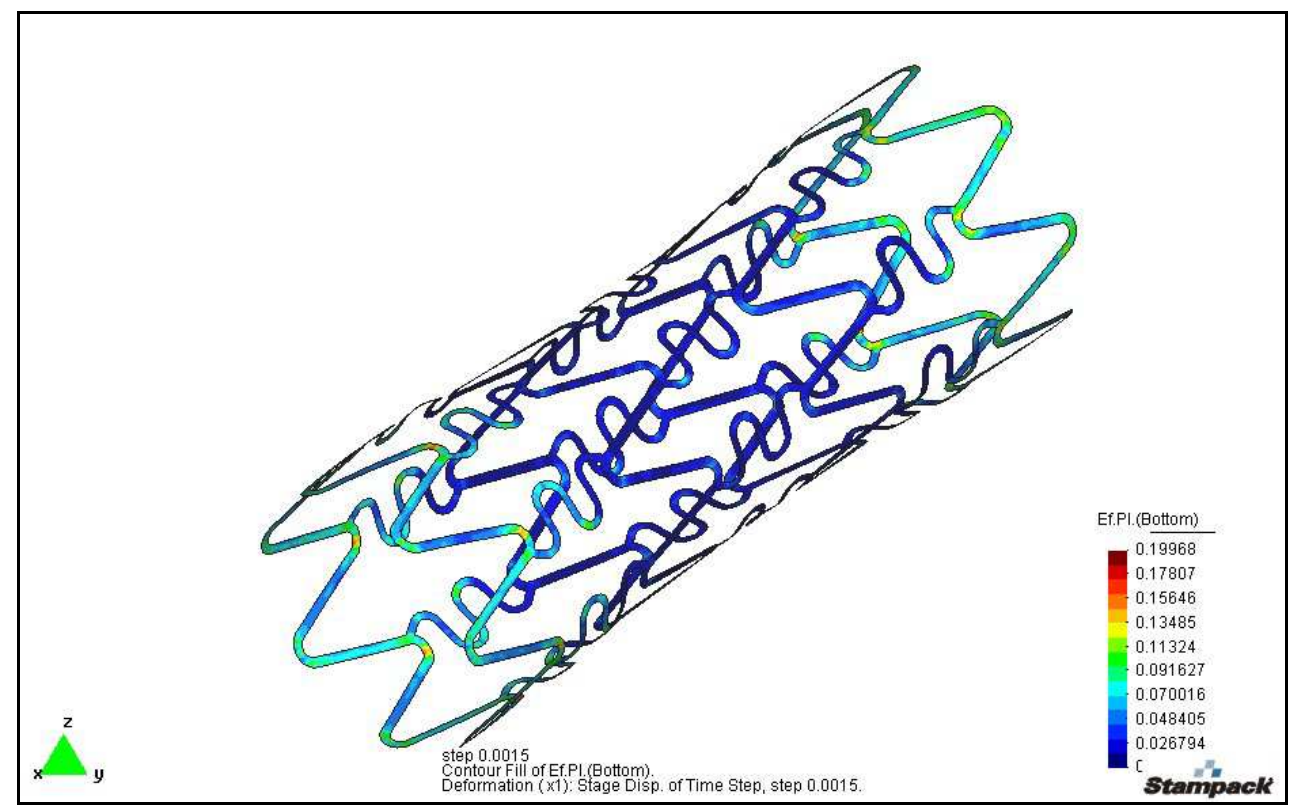

Figure 3. Final shape and plastic strain field from stent after the angioplasty simulation. 
The figure 4 shows the stent structure regions subjected to the wrinkling, thinning and buckling. The regions with blue color indicate the wrinkling caused by the compressive stress and the areas with red color represents the thinning due to the tensile stress. According to this figure, there is no risk of structural risk in the stent after the expansion. The reason of this is that the maximum level of the plastic strain has observed in the expanded stent is relatively low, approximately 19\%, as can be seen in the Fig. 3. Although this small plastic strain preserves the structural integrity from stent, the hardening of stent material caused by its expansion improves its stiffness and preserves the opened arterial wall.

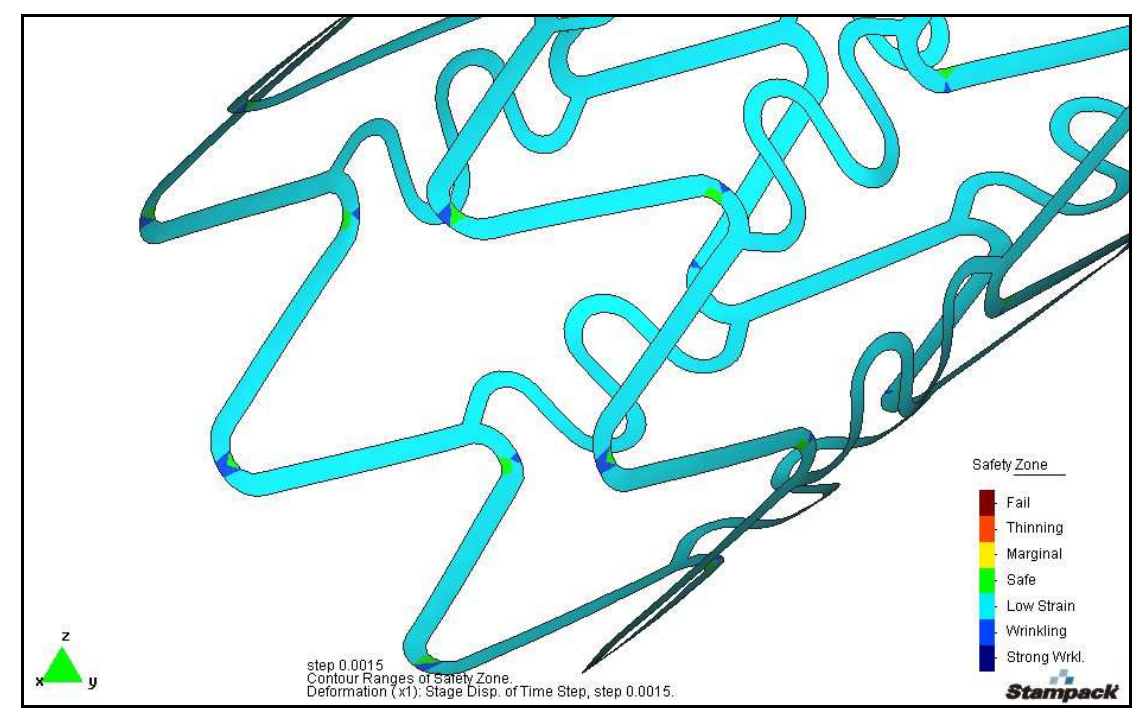

Figure 4. Safety zone of the expanded stent model.

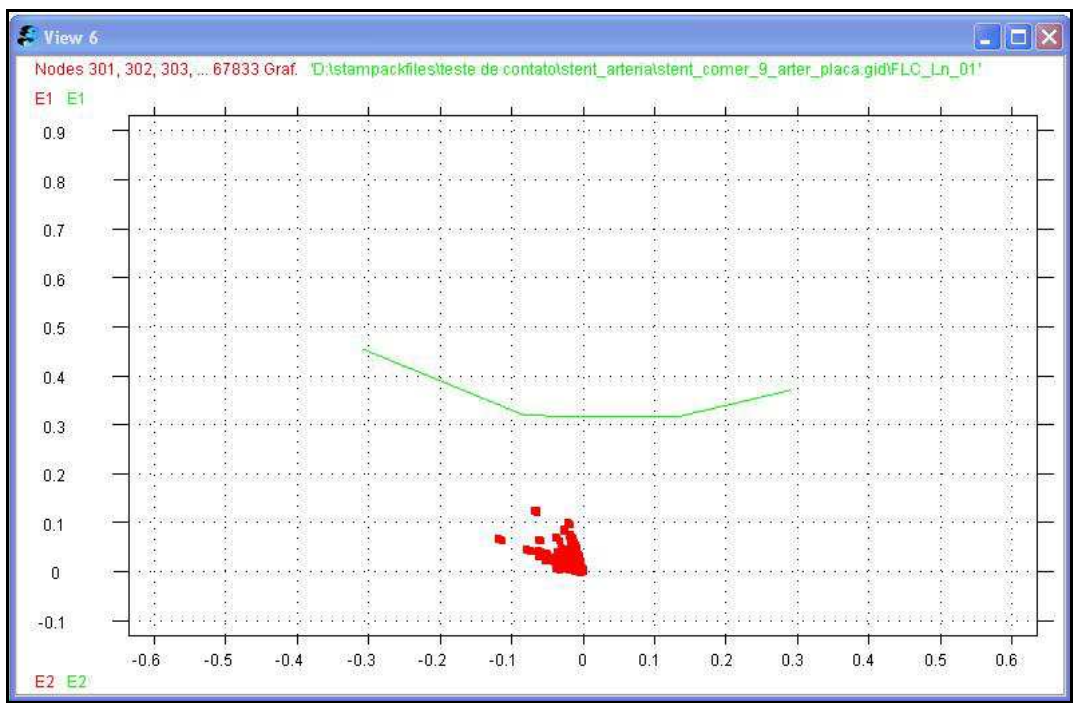

Figure 5. Forming Limit Diagram of the stent material after the expansion simulation. 
Another way of checking the structural integrity of the stent is from the Forming Limit Diagram (FLC) has illustrated in the Fig. 5. According to the FLC, all nodes from the stent finite elements model are below the Keeler-Goodwin diagram of the stent material. Thus, as the principal strain field has observed in this figure is not larger than the Keeler-Goodwin diagram, it is not expected risk of rupture or damage of in any node of stent finite elements model. In fact, with this analysis methodology, it is possible to predict if the stent will be successfully implanted in the artery by considering the dynamics effects caused by the applied pressure during the angioplasty procedure.

The figure 6 shows the final shape and the principal strain distribution in the artery and stenotic plaque. After the angioplasty, it is clearly observed that the expanded stent cause large strains in the arterial wall and plaque. It is expected that is no risk of rupture in the artery and stenotic plaque since their materials are hyperelastic. However, the high level of the stress (about 69\%) has observed in the arterial wall and plaque may cause hyperplasia and restenosis in stenosed artery. It is interesting to note that the maxima stress occur in the flexible links elements of the stent structure. During the angioplasty, these flexible links improve the capability of the set stent, catheter and balloon of moving inside the blood vessel with tortuosity. Nevertheless, for this particular stent design, the results proved that the flexible linkage elements increase the contact stress level in the artery and plaque wall.

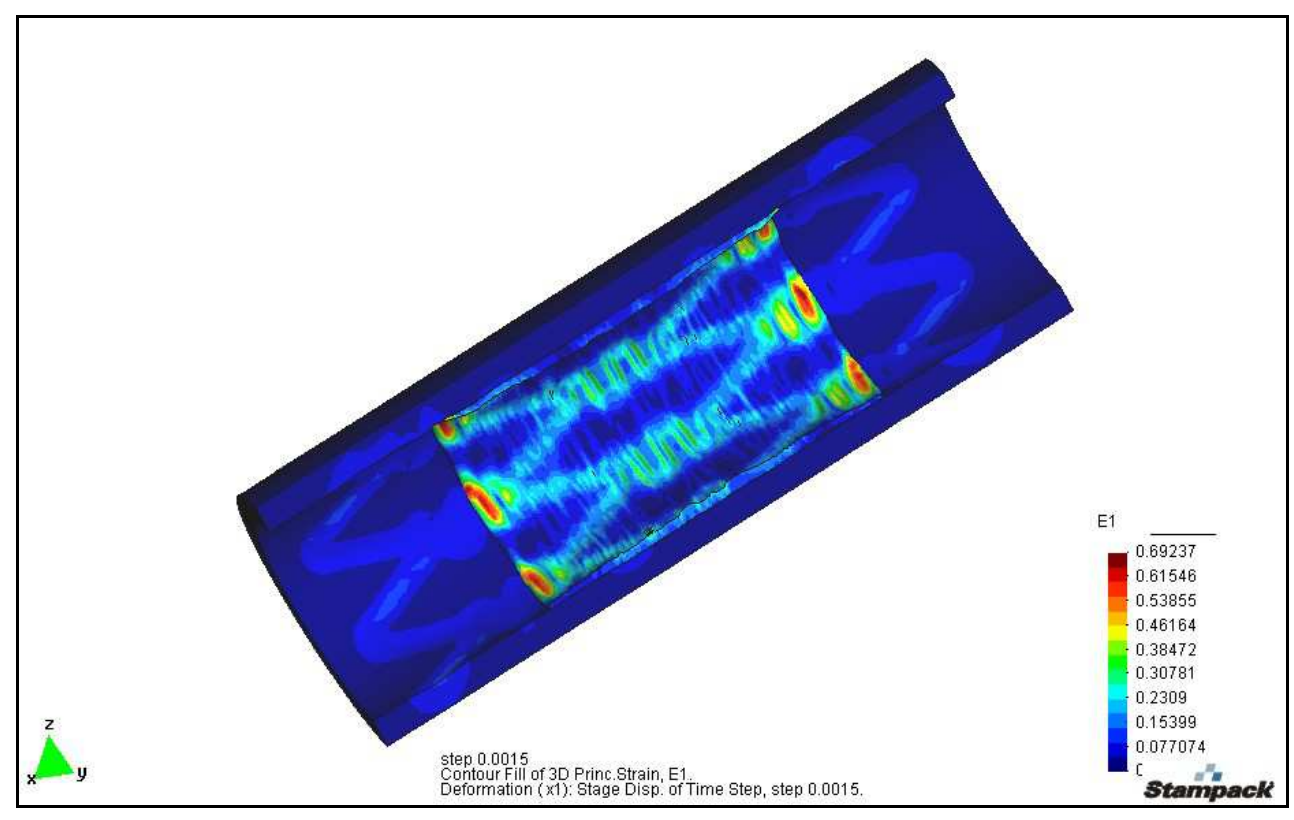

Figure 6. Principal hyperelastic strain field in the artery and stenotic plaque after the angioplasty simulation. 


\section{CONCLUSIONS}

In this work, it was purposed a methodology to study the structural integrity of stents and the interaction among the stent, artery and stenostic plaque during the angioplasty. For the simulation of the angioplasty procedure, it was used an explicit finite elements code based on the tube hidroforming process. An advantage of this methodology is that dynamic pressure applied on the internal surface from stent is also taken into account in the analysis. By using the plastic strain maps and the Forming Limit Diagram, it was possible to verify if the stent design has considered in this work has any risk of rupture after the angioplasty, as well as, if the artery and plaque are subjected to the high level of strains.

The stent geometrical model has studied in this work introduced a good performance according to the results obtained. After the expansion, it was not observed any region of the stent structure with strong wrinkling, thinning or buckling. In the Forming Limit Diagram, all points were far from the Keeler-Goodwin Diagram for the stent material. Furthermore, the expanded stent geometrical model has a good conformability, that is, a relatively high capability of absorb elastic strain energy from artery and plaque. On the other hand, it was verified the flexible links elements increased the hyperelastic strain level of the arterial wall and plaque. These contact stress caused by the expansion process of the stent may produce lesions at the artery and plaque wall and increase the risk of occurrence of hyperplasia and restenosis in the stenosed artery.

\section{Acknowledgements}

The authors would like to thank the CNPq for the financial support.

\section{REFERENCES}

[1] Lally C., Kelly D. J., Prendergast P. J., "Stents". Wiley Encyclopedia of Biomedical Engineering. 1-10, 2006.

[2] Lally C., Dolan F., Prendergast P. J., "Cardiovascular Stent Design and Vessel Stresses: a Finite Elements Analysis”. Journal of Biomechanics. 38, 1574-1581, 2005.

[3] Liang D. K., Yang D. Z., Qi M., Wang W. Q., "Finite Elements Analysis of the Implantation of a Balloon-Expanded Stent in a Stenosed Artery". International Journal of Cardiology. 104, 314-318, 2005.

[4] Stampack User Guide. "Sheet Forming and Stamping Analysis - Basic Concepts". Quantech ATZ. 2002.

[5] Batalha G. F., Abrantes J. P., Szabo-Ponce A., "Experimental and Numerical Simulation of Tube Hidroforming". Journal of Material Technology Processing. 164-165, 1140-1147, 2005. 
[6] Murphy G., "Similitude in Engineering”. Ed. Ronald Press Company, 1950.

[7] Onate E., Zarate F., Flores F., “A Simple Triangular Element for Thick and Thin Plate and Shell Analysis". International Journal of Numerical Methods in Engineering. 37, 25692582, 1994.

[8] Chua S. N. D., MacDonald B. J., Hashimi M. S. J., "Finite Elements Simulation of Stent and Balloon Interaction”. Journal of Material Technology Processing. 143-144, 591-597, 2003.

[9] De Beule M., Mortier P., Carlier G., Verhegghe B., VanInpe R., Verdonck P., "Realistic Finite Elements-Based Stent Design: The Impact of Balloon Folding". Journal of Biomechanics. 41, 383-389, 2008.

[10] Kumar R. K., Balakrishnan K. R., "Influence of Lumen Shape and Vessel Geometry on Plaque Stresses: Possible Role in the Increased Vulnerability of a Remodelled Vessel and the "Shoulder" of a Plaque". Heart. 91, 1459-1465, 2005. 